\title{
P-curving x-phi: Does experimental philosophy have evidential value?
}

\begin{abstract}
In this article, we analyze the evidential value of the corpus of experimental philosophy (x-phi). While proponents of $\mathrm{x}$-phi claim that its studies provide insight into philosophical problems, some philosophers and psychologists have expressed concerns that the findings from these studies lack evidential value. One reason for this could be selection bias (i.e., the selective publication of significant results) and p-hacking, where 'p-hacking' refers to the research practices that increase the odds of obtaining a p-value below the significance level. To find out whether the significant findings reported in x-phi papers result from selection bias or p-hacking, we applied a p-curve analysis to a corpus of $365 \mathrm{x}$-phi chapters and articles. Our results suggest that this corpus has evidential value, although there are hints of $\mathrm{p}$-hacking in a few parts of the $\mathrm{x}$ phi corpus.
\end{abstract}

\section{Introduction}

'Experimental philosophy' (henceforth 'x-phi') refers to the use of experimental tools to investigate questions that bear on philosophical issues. This approach remains controversial on several grounds. One challenge is that the findings of x-phi are produced unreliably, and have therefore little evidential value. In particular, one might worry that x-phi research suffers from questionable research practices, including the selective reporting of statistically significant findings and p-hacking. If this were the case, many of the effects experimental philosophers claim to have identified would likely be false positives and could not be justifiably brought to bear on any philosophical issue. 
In this paper, we examine whether the corpus of $x-p h i$ suffers from selection bias and $p$ hacking by employing what is called a p-curve (Simonsohn, Nelson, and Simmons 2014a). A pcurve is a distribution of statistically significant $p$-values for a corpus of experimental studies. The way the p-curve deviates from the uniform distribution indicates whether it was likely to have been the product of selection bias and p-hacking (more on this below).

We developed a dataset of 365 published chapters and articles, which includes nearly all x-phi studies written in English up to the year 2016. We then applied a number of p-curves to the corpus as a whole and to sections of this corpus. Our results suggest that $\mathrm{x}$-phi findings as a whole do not result from selection bias or p-hacking and thus that, overall, the corpus has evidential value. We also find that p-hacking may have occurred in a few subsets of this corpus and that $\mathrm{x}$-phi has methodologically improved.

Here is how we will proceed. In Section 1, we motivate our project to a greater extent. In Section 2, we describe p-curves in more detail. We report our methods and results in Section 3. We discuss the significance of our findings in Section 4.

\section{Motivation}

Challenges to the relevance of x-phi findings can be divided into two categories: conceptual and empirical challenges. Conceptual challenges include arguments to the effect that, regardless of the quality of $\mathrm{x}$-phi studies, their findings are not relevant to philosophical theorizing. This might be due to a misunderstanding of philosophical methodology or the nature of philosophical argumentation (Cappelen 2012; Deutsch 2015; Ludwig 2018; Stuart 2015; for discussion of some of these arguments, see Colaço and Machery 2017; Machery 2017). Included in this category are challenges that the participants in x-phi studies, typically but not exclusively lay 
people, do not have the right expertise or exhibit the appropriate reflectivity to judge philosophical cases (Ludwig 2007, 2018; Kauppinen 2007; though see Colaço, Kneer, Alexander, and Machery, ms; Machery 2017; Stich and Tobia 2018). Empirical challenges, by contrast, relate to how x-phi studies are designed and their results analyzed. These include issues with experimental design (Cullen 2010; Woolfolk, 2011, 2013; Scholl, ms) and reported replication failures (Seyedsayamdost 2015a, b; Kim and Yuan 2015; Adleberg, Thompson, and Nahmias 2015; Machery et al. forthcoming), which suggest problems with the studies that experimental philosophers have conducted. Woolfolk, for instance, asserts that 'rather ironically, the experiments conducted by experimental philosophers frequently fail to meet the methodological standards that are articulated by the experts on research design in those fields they would emulate' (2013: 80). Empirical challenges raise in-practice, as opposed to inprinciple doubts about the relevance of the findings of $\mathrm{x}$-phi to philosophical discussion.

While the methodological concerns one might have about $\mathrm{x}$-phi are manifold, here are two specific reasons to worry that $\mathrm{x}$-phi experimenters may have inadvertently engaged in $\mathrm{p}$ hacking. First, the majority of x-phi studies use the experimental methods of social psychology, a field that has recently faced allegations of p-hacking (Simmons et al. 2011), one of the most likely sources of its current 'replication crisis' (Open Science Collaboration 2015). Given that Xphi inherits many of its experimental and statistical practices from social psychology, there is reason to worry that it may suffer from the same problem. In this spirit, Vazire (2015: 46) notes that some of the common practices in psychology 'increase the chances of producing false, unreplicable results. These practices have come to be called 'questionable research practices' (QRPs), or 'p-hacking,' but it is important to note that most were not considered questionable by many until recently, and some are still taught in textbooks and research methods courses'. Thus, 
in her view, 'the most important thing experimental philosophers can learn from psychologists is to avoid these practices, follow the new 'best practices,' and spare themselves the decades of growing pains that psychology has experienced' (Vazire 2015: 46). Second, x-phi has been performed by individuals who are professionally trained as philosophers, and who may therefore have limited experience as empirical researchers (Williamson 2010). This has led to speculation that experimental philosophers may lack the requisite training in statistics and experimental design, leading them to engage in research practices they do not recognize as questionable (Woolfolk 2011, 2013).

Recent work assuages to some extent the concerns about the quality of methodological practices in experimental philosophy. The XPhi Replicability Project (Cova et al. 2018) set out to replicate $40 \mathrm{x}$-phi studies, some of which were chosen based on their citation number, while others were chosen at random. The results of this project indicate that $\mathrm{x}$-phi findings exhibit much greater reproducibility than those in fields like social psychology.

While the XPhi Replicability Project has numerous virtues, it is also limited in important respects. First, only a small subset of x-phi studies were targeted for replication, and these studies were not entirely chosen at random. Because of this, there is a worry about the representativeness of the studies that were targeted. Our project has a much larger dataset, which is more representative of the field of $\mathrm{x}$-phi. Thus, we can determine the evidential status of the research corpus as a whole. Further, because of the small number of studies included in Cova et al. (2018), claims about parts of the corpus of experimental philosophy cannot be made with confidence. By contrast, the number of reported studies we have included in our dataset allows us to investigate facets of this corpus in more detail. This allows us to investigate changes in the 
quality of published studies over time, across research questions, philosophical subfields, and more.

Colombo and colleagues (2018) have examined the quality of the methods in experimental philosophy from another angle. Recent work suggests that inconsistencies in statistical reporting (that is, the frequency of discrepancies between the reported parameters of statistical tests and the reported p-values) are surprisingly common in psychology (Bakker and Wicherts 2011; Nuijten, Hartgerink, van Assen, Epskamp, and Wicherts 2016). Colombo et al. examined the rate of inconsistencies in statistical reporting in $220 \mathrm{x}$-phi studies and found that it is lower than in psychology, meaning that in this respect at least x-phi is in better shape than psychology.

While the findings from Colombo and colleagues' analysis are valuable, their results are consistent with rampant $\mathrm{p}$-hacking, and thus cannot exclude $\mathrm{p}$-hacking in $\mathrm{x}$-phi, something that the p-curve can do. Colombo and colleagues also p-curved their results, but their sample is smaller than ours and they did not p-curve various subsections of their sample, as we do below.

\section{P-Curve Analysis}

P-curving was developed as a strategy to examine the evidential value of a corpus of studies, by determining if the distribution of significant p-values of their main reported results is indicative of selection bias and p-hacking (Simonsohn, Nelson and Simmons 2014a, b; Simonsohn, Simmons and Nelson 2015). P-curve analysis has already been used to identify several corpora in psychology and neuroscience that suffer from p-hacking (e.g., Segerstrom and Miller 2013; Simonsohn, Nelson and Simmons 2014a; Vadillo, Gold and Osman, 2016; Medina and Cason, 
2017; Simmons and Simonsohn 2017) as well as to provide evidence that some corpora do have evidential value (e.g., Mahowald, James, Futrell and Gibson 2016).

P-curve analysis works as follows. If all the null hypotheses tested by a set of studies are true, then the corresponding p-values should be uniformly distributed, and the p-curve for such a set of studies should look like Fig. 1A. On the other hand, if the null hypotheses are false, then we expect the distribution of p-values to be right-skewed: roughly, there should be fewer high pvalues (near to .05) than low p-values. In this case, the p-curve distribution should look like Fig. 1B. The more powerful the tests are, the more right-skewed the distribution of $\mathrm{p}$-values.

So, we can test whether a set of studies has evidential value by testing whether the distribution of significant p-values in a set of studies differs from the uniform distribution. If we can reject the null hypothesis that the p-curve is uniform, we can conclude that the set of studies under scrutiny has evidential value as a group. That is, at least some of the studies in this set (but not necessarily all) correctly reject false null hypotheses. Thus, the published p-values cannot merely be the result of a selection bias, i.e., the selective publication of false positives.

A p-curve analysis could fail to reject the null hypothesis that the p-curve is uniform for one of two reasons: the null hypotheses tested by a set of studies happen to be true or the power of the test is too low. To distinguish these two hypotheses, p-curve analysis introduces a distribution of $\mathrm{p}$-values resulting from tests with a low power (.33). If the p-curve is significantly flatter than this distribution, one concludes that the set of p-curved studies has no evidential value: either the null hypotheses are true, or the effects are too small to be measured (for a discussion of this use of power analysis, see Machery 2012). If we cannot reject the null hypothesis that the $\mathrm{p}$-curve is the distribution of $\mathrm{p}$-values resulting from tests with a .33 power, 
we infer that our analysis has not enough power to conclude that a literature has no evidential value.

Furthermore, researchers who are p-hacking, e.g., who are collecting data until they obtain statistical significance and then stop (a practice called 'optional stopping'), are trying to push p-values below the significance level, set by convention at .05 . If researchers are p-hacking, we should then expect a larger number of p-values just at or below .05 than what would be expected by chance. P-hacking will thus produce a p-curve that is left-skewed, as in Fig. 1C.

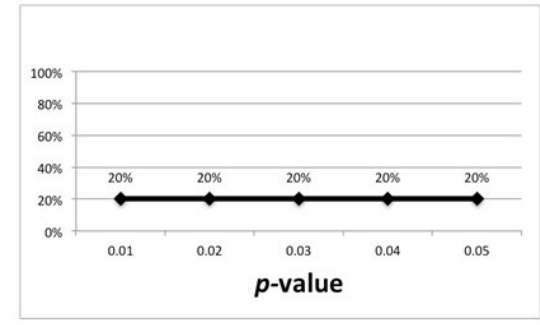

A

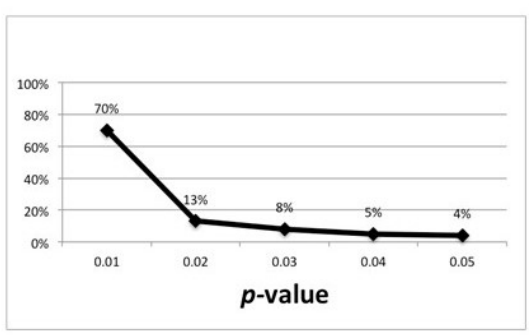

B

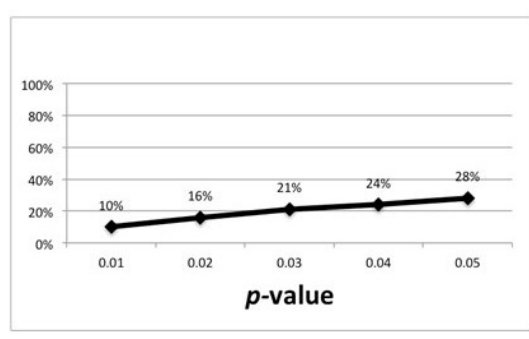

C

Fig. 1. Different p-curves. A: p-curve with uniform distribution. B: a 'right-skewed' p-curve, displaying evidential significance. C: a 'left-skewed' p-curve, displaying evidence of p-hacking.

A set of studies will only have the p-curve shape in Figure 1C if the null hypothesis is true and there is intense $\mathrm{p}$-hacking. For any non-zero effect size, the p-curve will be right-skewed, at least to some extent. Still, the shape of the p-curve can tell us how confident to be that a set of studies contains results that were $\mathrm{p}$-hacked. For example, a U-shaped p-curve exhibits both a right and a left skew, indicating both a real effect and p-hacking. As Head et al write, if 'researchers p-hack when there is a true effect, the p-curve will be exponential with right skew but there will be an 
overrepresentation of p-values in the tail of the distribution just below 0.05' (Head et al. 2015: 3; see also Simonsohn, Nelson and Simmons 2014a).

Finally, p-curve analysis can be applied to sets of studies investigating either a single null hypothesis or different null hypotheses. Thus, we are not limited to p-curving only the studies that investigate a hypothesis about a single effect.

\section{Materials and Methods}

\section{Dataset Coding}

X-phi studies were identified by searching philpapers.org, Google Scholar, online xphi bibliographies (including the Stanford Online Encyclopedia), and searching individual journals (including Review of Philosophy and Psychology, Mind and Language, Philosophical Psychology, Analysis, Nous, Philosophy and Phenomenological Research, Philosophers' Imprint, Synthese, Psychology, Cognition, Cognitive Science and others). Three different coders were then trained to identify the date published (online first, when different dates existed), whether the paper was a replication or not, chapter or article, the number of authors, whether any of the authors was a non-philosopher, what area of philosophy the paper fit into, the number of studies in the paper, and all the test statistics. The coders agreed on 92.6 percent of studies (excluding disagreements irrelevant to the p-curve). Entries that did not match were corrected by the authors of this paper.

\section{Data Collection}


The corpus includes 365 works that were published between the years of 1997 and 2017. Data collection was terminated in 2016, as our aim was to investigate three five-year intervals between 2001 and 2016. Data from 2017 is not exhaustive; only works that were forthcoming in 2016 and published in 2017 are included. Data were collected from English-language publications only.

\section{Inclusion Criteria}

To be included in our dataset, three criteria had to be met. First, the work had to include as author or co-author at least one individual with a PhD in philosophy or a cognate discipline (e.g., history and philosophy of science) and possess an affiliation to a philosophy department. This excludes works authored by psychologists or neuroscientists alone, even when philosophically relevant. Second, the work must either report novel data or analyze data previously collected by other philosophers in a new way. This excluded replication articles, or works where philosophers analyzed data collected by non-philosophers. Third, the work must be explicitly framed as bearing on a philosophical issue. This excludes scientific works that happen to include collaborators that are philosophers (e.g., some work in the philosophy of science in practice).

\section{Determination of main statistic(s) for a study}

We recorded the results of both main and auxiliary statistical tests in each study. Main statistics were determined by relating them to the main hypotheses presented in each work. There were often more than one main statistic for a given study, and more than one study per paper. All other statistics were reported as 'other' in the dataset. P-curves are run on the main statistics only, because we are interested in the evidential status of the main conclusions (the main hypotheses 
tested). Because the p-values in the p-curve must be independent, we only used the statistic coded as the main statistic for our p-curves.

\section{Designation of Field of Philosophy}

We divided the corpus into eight categories in order to assign each of the 365 articles to a single field of philosophy. The categories were metaphysics, epistemology, philosophy of action, philosophy of mind, ethics, philosophy of language, philosophy of science, and 'other'. Papers in the 'other' category belong to fields that are less well-represented in x-phi, like philosophy of religion, race, aesthetics, medicine, and metaphilosophy. Categorization was based on the topic of the paper. Categorization was not based on operational criteria, but on our own sense of the field in which the papers belong. Some decisions were made in advance of our categorization. For example, we had decided to categorize work on intentionality and free will into philosophy of action, given that this field is traditionally where these topics are investigated.

\section{Negative versus Positive Program}

We divided the corpus into the so-called 'negative' and 'positive' programs. The negative program includes all papers meant to undermine traditional philosophical methods such as the method of cases (O’Neill and Machery 2014; Machery 2017), the positive program everything else. Categorization was not based on operational criteria, but on our own sense of the program in which the papers belong.

\section{P-Curve App}


The p-curves were generated using version 4.06 of the p-curve app, created by Simonsohn, Nelson, and Simmons. The app can be accessed here: http://www.p-curve.com/app4/, and its Rcode here: http://p-curve.com/app4/pcurve_app4.06.r. For more details on the statistical underpinning of the app, or the logic supporting the statistics, see Simonsohn, Nelson and Simmons $(2014 \mathrm{a} / \mathrm{b})$.

\section{Results}

The skews of our $\mathrm{p}$-curves provide insight into which areas of $\mathrm{x}$-phi have findings that cannot be explained by selection bias or p-hacking. In each figure, the solid line is the observed p-curve, which is indicative of the corpus or relevant corpus subset. The hatched lines are the uniform distribution of $\mathrm{p}$-values (narrow hatches) or the distribution of $\mathrm{p}$-values resulting from a lowpowered (.33) test (wide hatches).

Overall 


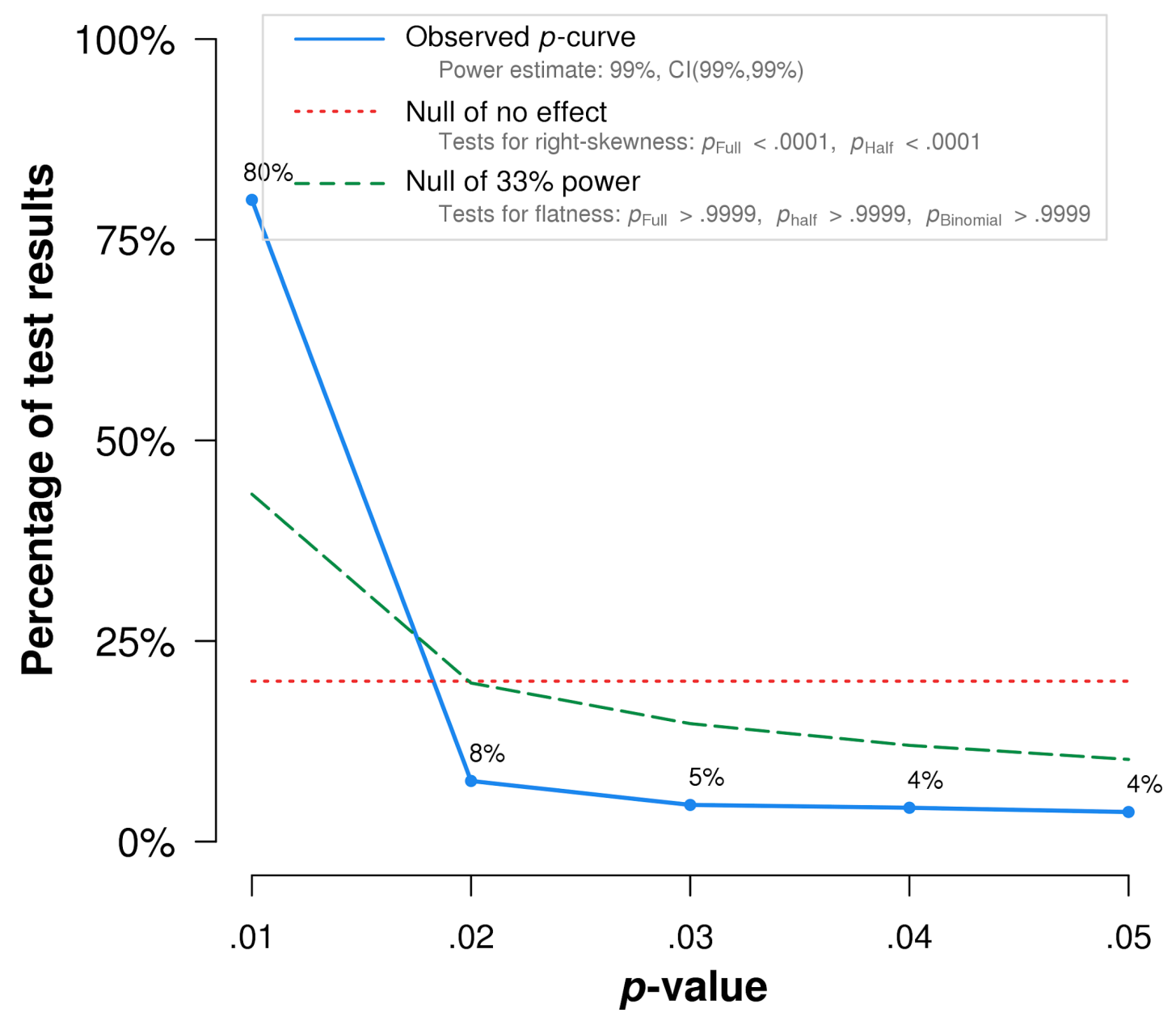

Note: The observed $p$-curve includes 569 statistically significant $(p<.05)$ results, of which 516 are $p<.025$. There were 27 additional results entered but excluded from $p$-curve because they were $p>.05$.

Fig. 2. P-curve for x-phi corpus as a whole.

The overall p-curve for the corpus of $\mathrm{x}$-phi is right-skewed.

X-Phi Results over Time 
We then examined the corpus by periods in order to see whether the frequency of p-hacking changes over time. We have divided the corpus into three intervals: early, which includes all the articles and chapters published before 2006, middle, which includes those published from 2006 to 2010, and later, which includes those published in 2011 up to the most recent in our corpus. These intervals do not correspond to any transformation or discontinuity in x-phi, but they give us three groups of five or more year intervals, to determine how the field has changed over time, if at all.

The p-curve for the early x-phi has a U-shape, unlike that for the corpus as a whole (Figure 3). The right skew is more pronounced for the middle x-phi (Figure 4), and even more so for the later x-phi (Figure 5). Correspondingly, the frequency of high p-values is lower in the middle xphi and even lower for the later x-phi. 


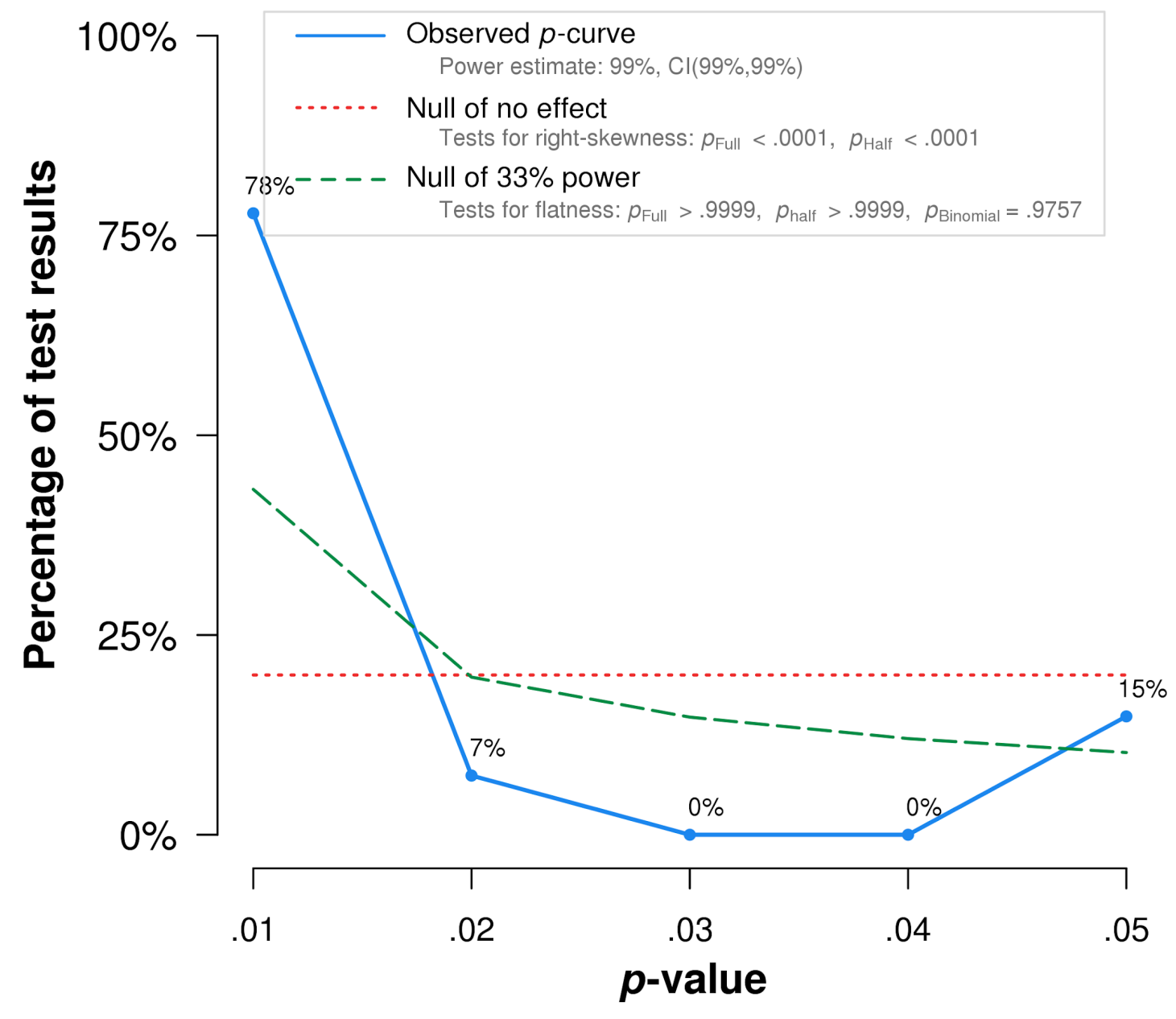

Note: The observed $p$-curve includes 27 statistically significant $(p<.05)$ results, of which 23 are $p<.025$. There were 15 additional results entered but excluded from $p$-curve because they were $p>.05$.

Fig. 3. P-curve for studies published up to but not including 2006. 


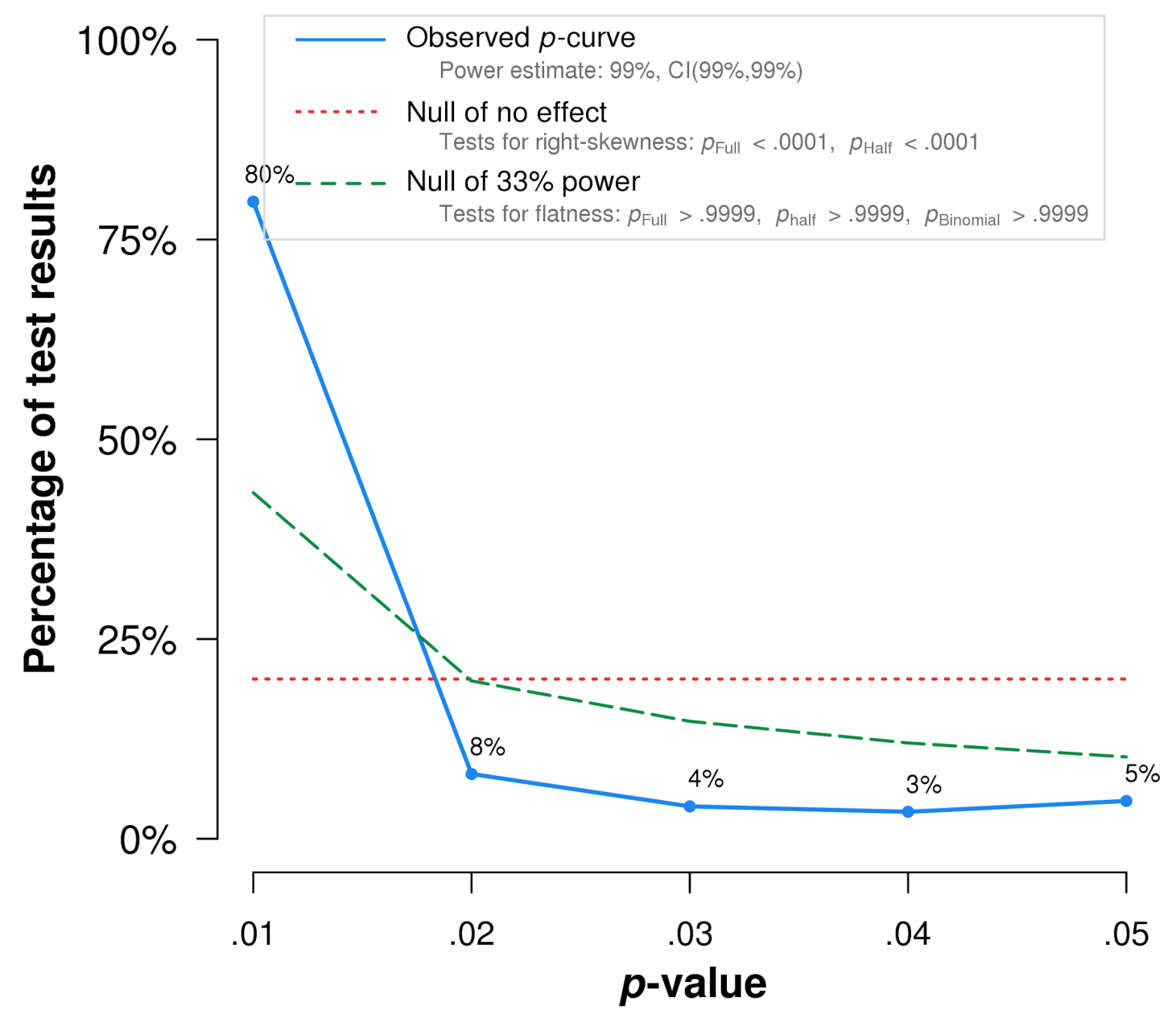

Note: The observed $p$-curve includes 148 statistically significant $(p<.05)$ results, of which 136 are $p<.025$. There were 4 additional results entered but excluded from $p$-curve because they were $p>.05$.

Fig. 4. P-curve for the studies published in 2006 up to but not including 2011. 


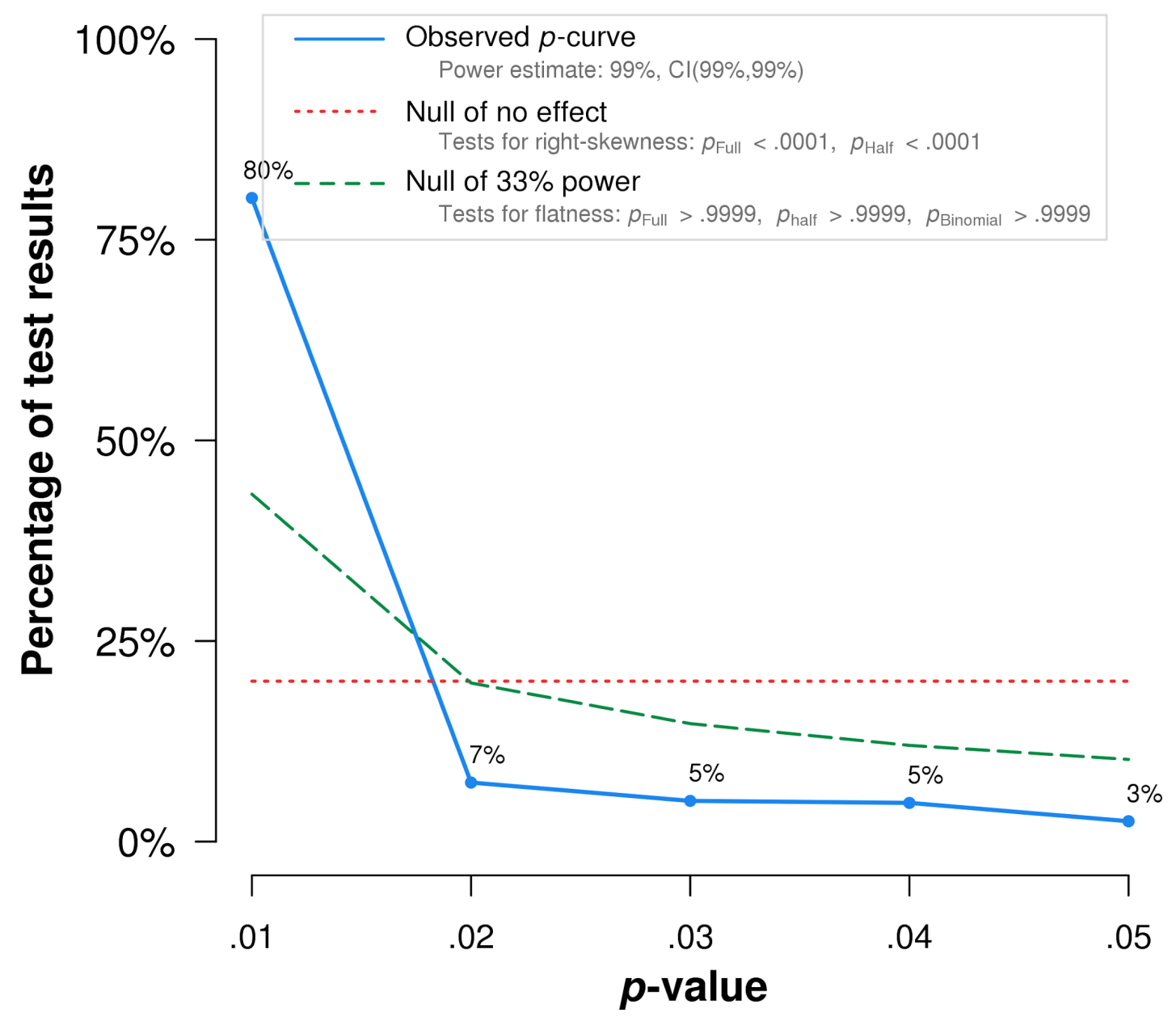

Note: The observed $p$-curve includes 394 statistically significant $(p<.05)$ results, of which 357 are $p<.025$. There were 8 additional results entered but excluded from $p$-curve because they were $p>.05$.

Fig. 5. P-curve for the studies published in 2011 up to the most recent studies in our dataset.

Field of Philosophy

The p-curve for each field of philosophy has a right skew (Figure 6). However, the p-curve for ethics has a tail of high p-values. 


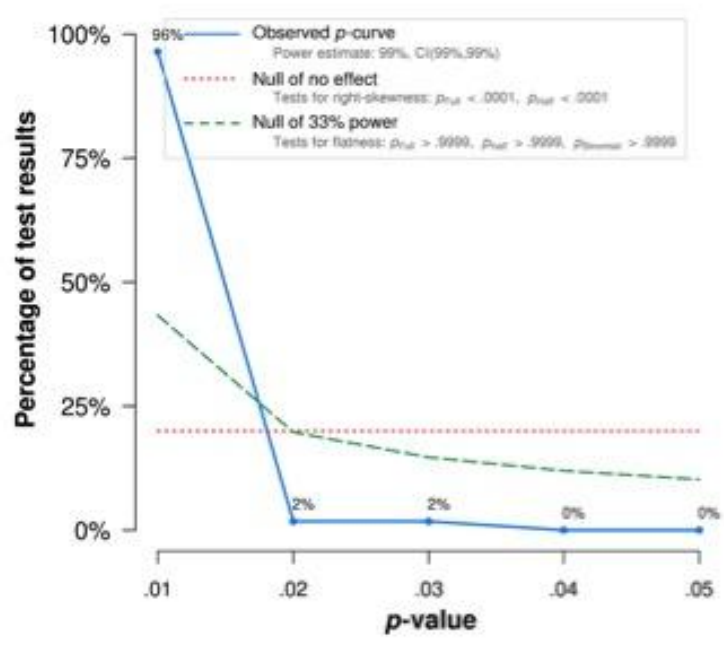

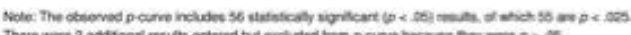

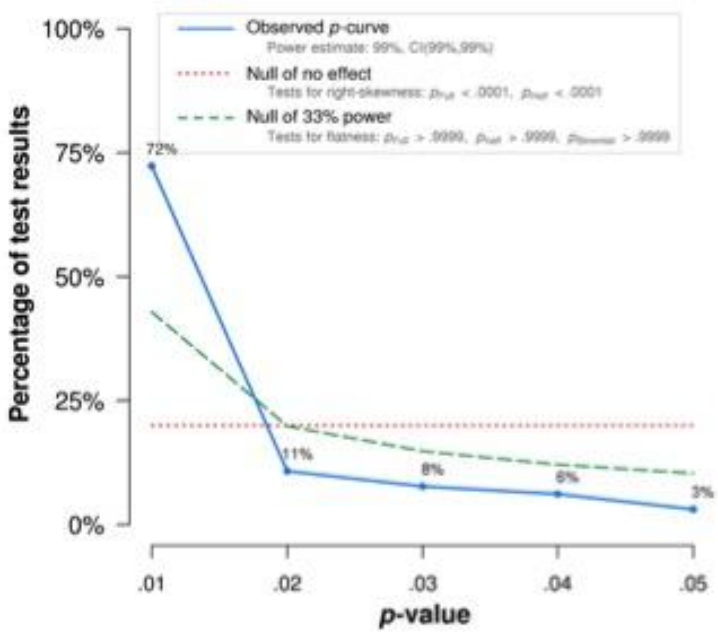

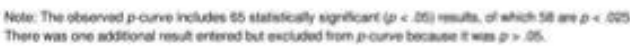

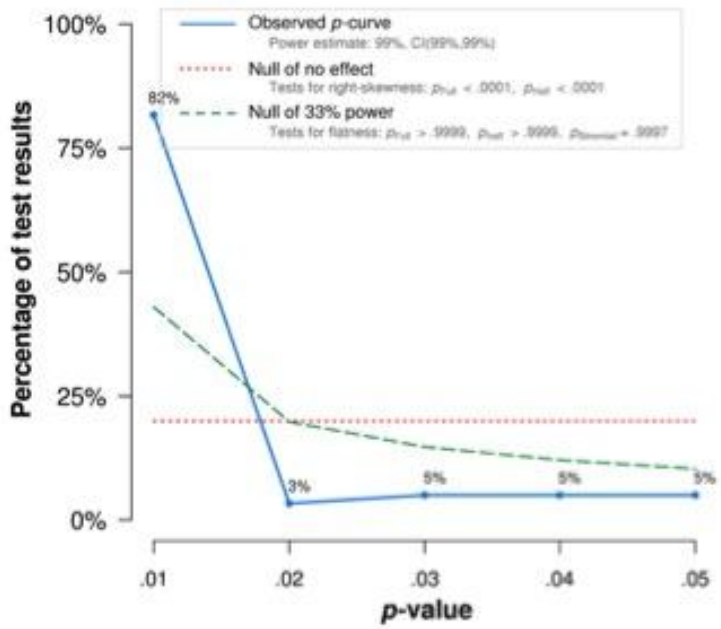

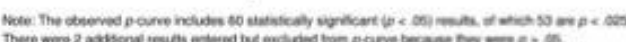

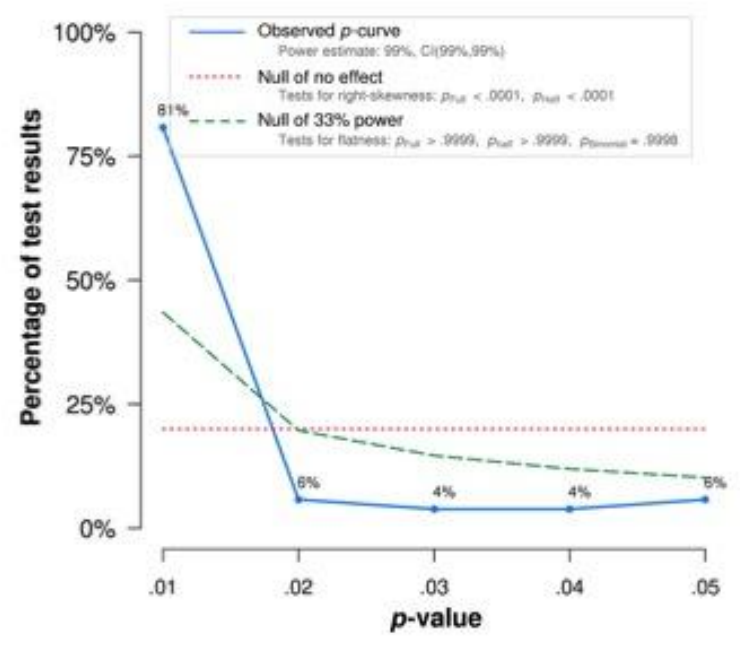

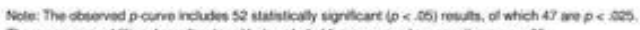

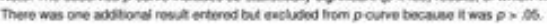

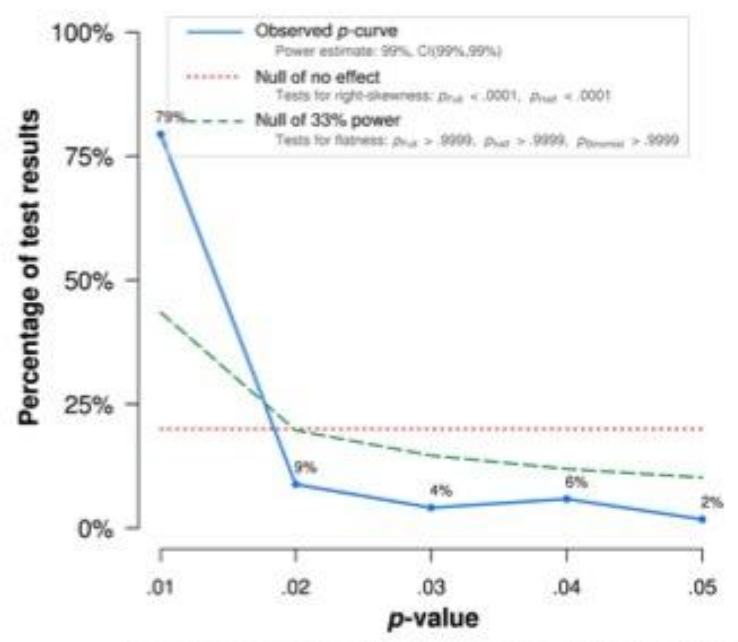

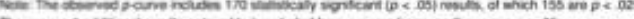

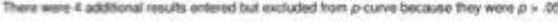

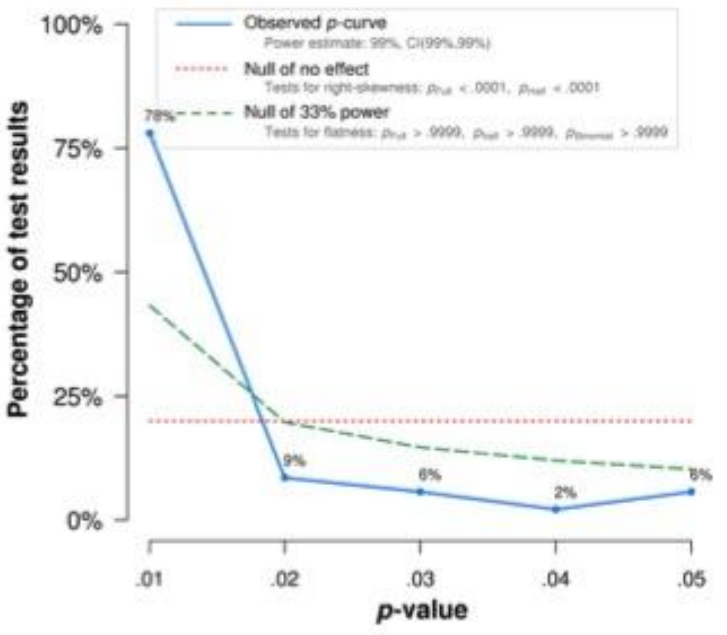

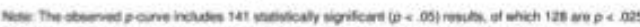
Dero 
Fig. 6. P-curves for the fields of philosophy: metaphysics (top left), epistemology (top right), language (middle left), action (middle right), mind (bottom left), and ethics (bottom right).

\section{Collaborators}

The p-curves for papers written by philosophers only as opposed to philosophers in collaboration with non-philosophers are both right-skewed, and there are no important differences between them (Figure 7).
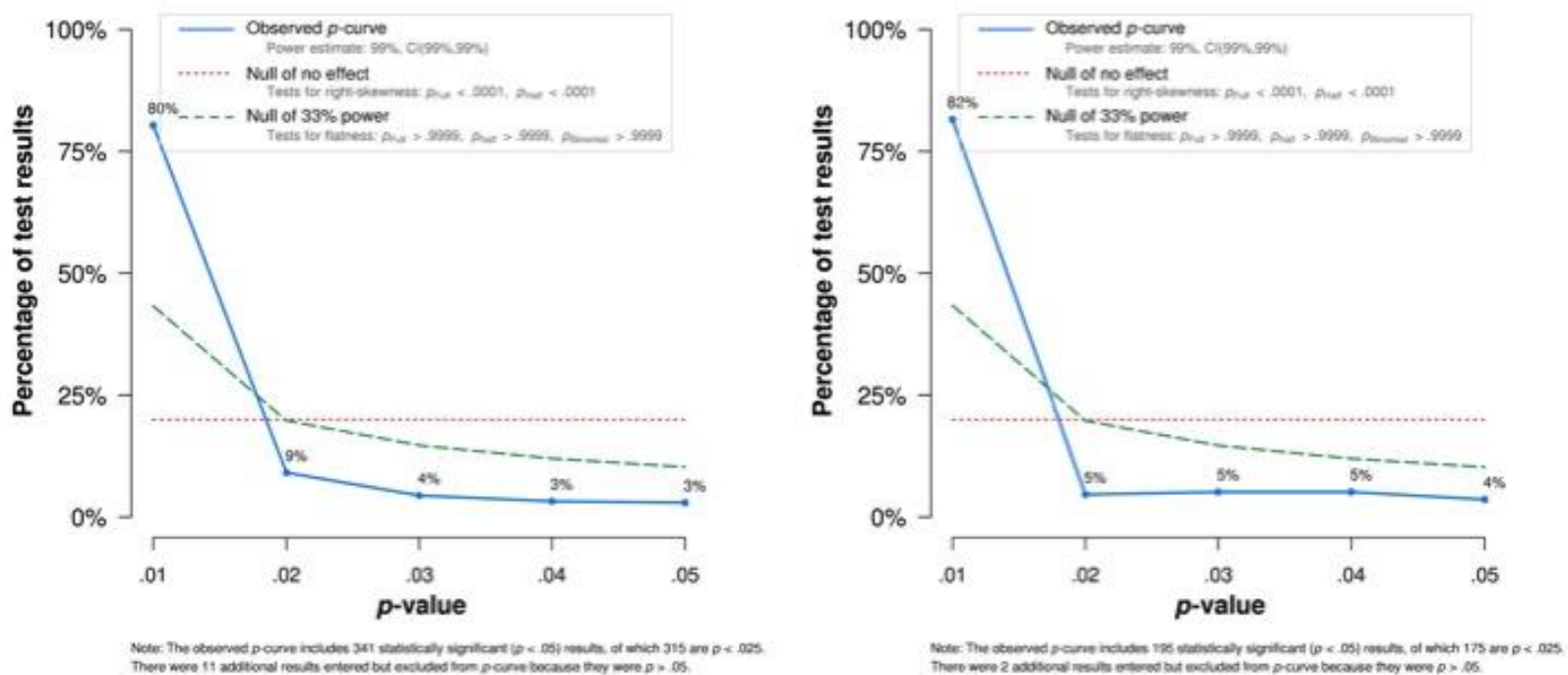

Fig. 7. P-curve for the studies involving only philosophers (left) and philosophers in collaboration with non-philosophers (right).

\section{Small Sample Sizes}

The p-curve for the studies with a sample size less than 20 is right-skewed, but has also a large tail of high p-values (Figure 8). 


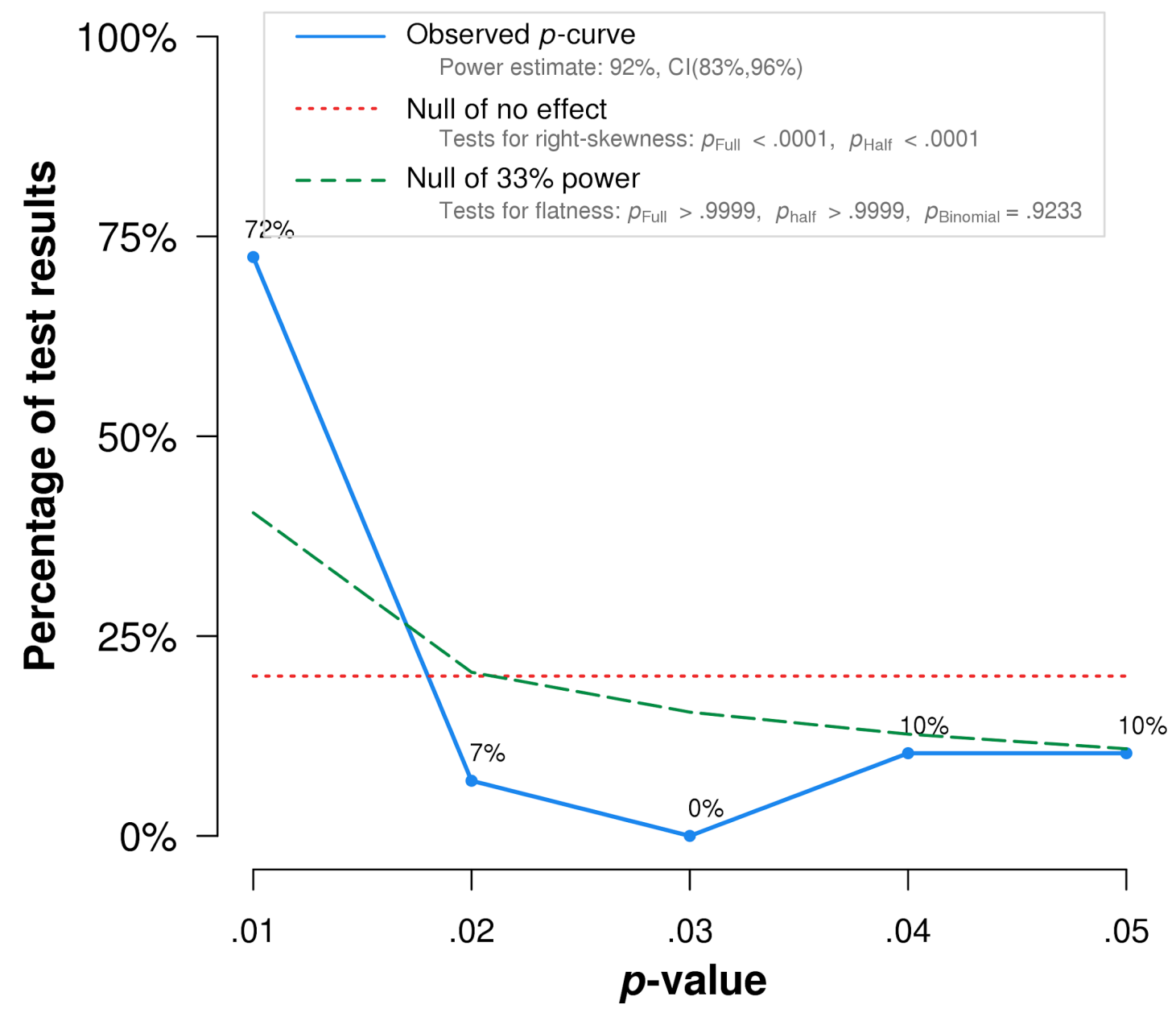

Note: The observed $p$-curve includes 29 statistically significant $(p<.05)$ results, of which 23 are $p<.025$. There were no non-significant results entered.

Fig. 8. P-curve for the studies with a sample size less than 20.

For comparison, here is the p-curve for the studies with a sample size between 500 and 1000: 


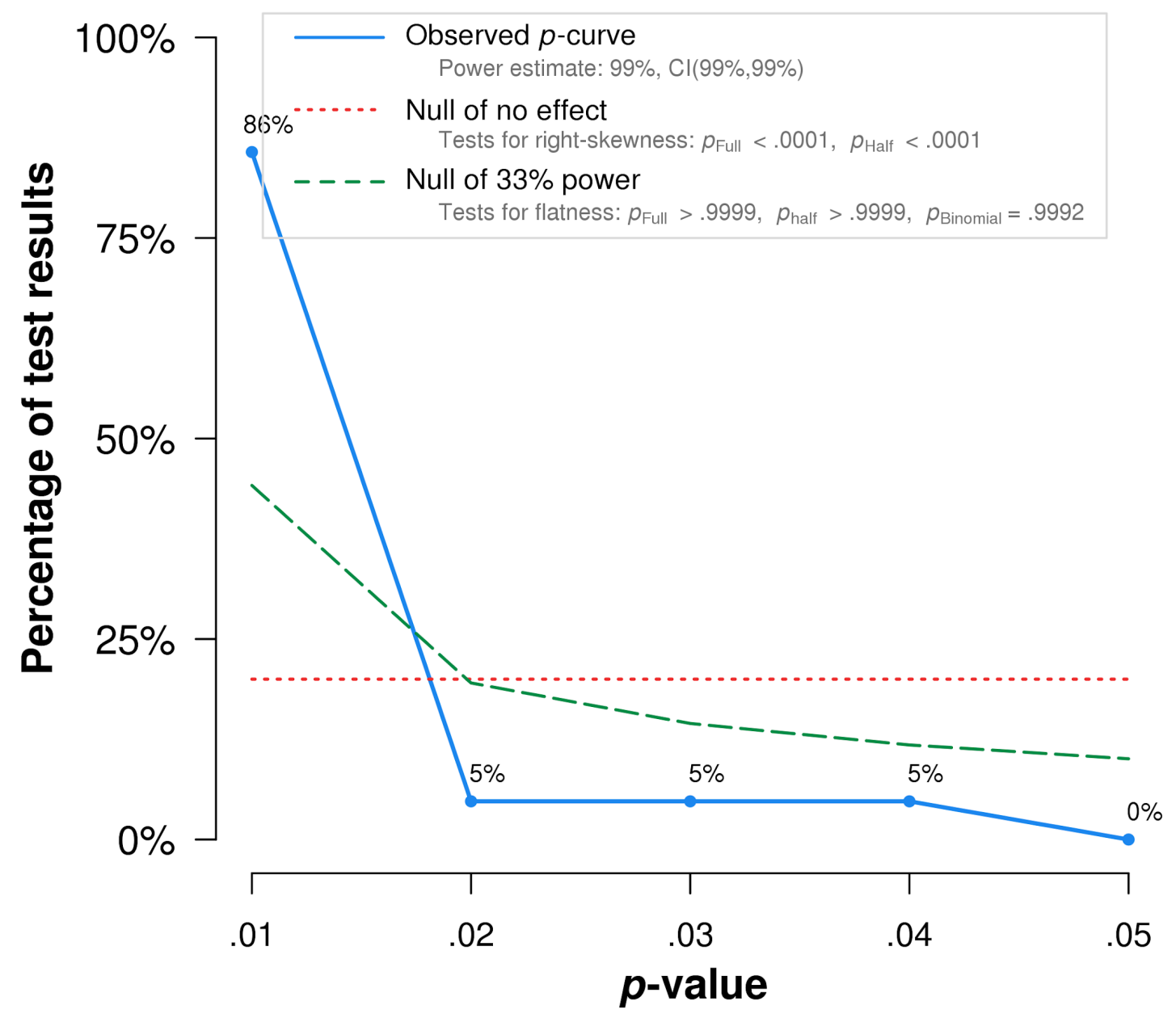

Note: The observed $p$-curve includes 21 statistically significant $(p<.05)$ results, of which 20 are $p<.025$. There were no non-significant results entered.

Fig. 9. P-curve for studies with sample size between 500 and 1000

Negative Versus Positive X-Phi

Negative x-phi and positive x-phi both have a right skew. Positive x-phi's p-curve has a high number of high p-values not present in the negative x-phi p-curve (Figure 10). Also the proportion of low p-values is higher in the negative $\mathrm{x}$-phi corpus. 

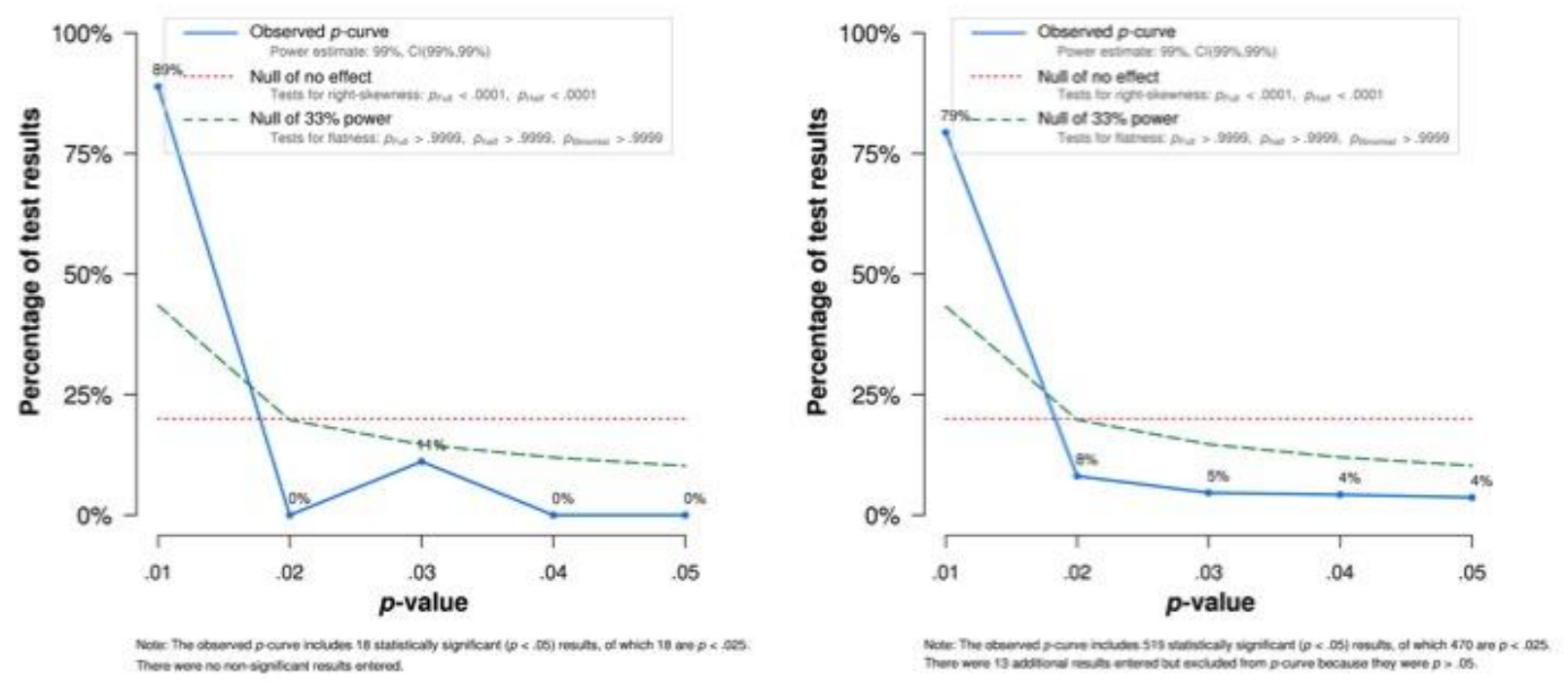

Fig. 10. P-curve for negative x-phi (left) and positive x-phi (right).

In sum, all p-curves reported in this paper are right-skewed, although some $\mathrm{p}$-curves have high $\mathrm{p}$ value tails. Both of these can be taken into account when assessing the evidential value of the corpus, and the explanatory adequacy of selection bias and p-hacking for reported significant results.

\section{Discussion}

Overall, the x-phi corpus fares well when analyzed with the p-curve. The right skew of $\mathrm{p}$-values for the main statistics in the corpus (Figure 2) suggests that, whatever selection bias and phacking might have occurred in the field, they are not an adequate explanation for the results reported in the corpus as a whole. Thus, the corpus of $x$-phi results has evidential value.

Whatever other concerns one may have about the relevance of x-phi studies for philosophical problems, it cannot be dismissed by alleging that it suffers from the questionable research practices to which the p-curve is sensitive. 
When our corpus is broken down into subsets, we see evidence of p-hacking in some of the resulting subsets. The p-curve for the years 2001-2005 (Figure 3) is somewhat skewed to the left, as there are a large number of tests with p-values just below .05 . P-hacking probably explains some of the results reported by the first x-phi studies, although the high number of low p-values nevertheless suggests that many of the investigated effects are real. The situation improves in the p-curve for the years 2006-2010 (Figure 4), with a greater right skew and a lessened left skew, suggesting that many of the effects investigated by experimental philosophers are real and that $p$-hacking is less common. The p-curve for the most recent years (2011-2017) is properly right skewed and shows no trace of p-hacking (Figure 5). We conclude that experimental philosophers have identified real effects in many of their studies, and that their empirical work has methodologically improved. The prominence of p-hacking in the corpus appears to diminish with time. This may be due to experimental philosophers becoming more aware of the perils of questionable research practices, or else designing their experiments with greater care (e.g., by relying less on optional stopping).

Turning now to the areas of philosophy that we distinguished, metaphysics, language, action, and epistemology all have evidential value (Figure 6). Ethics, though right-skewed, has a tail of high p-values, suggesting that some work in experimental ethics suffers from p-hacking (Figure 6, bottom). Negative x-phi comes out looking better than positive x-phi: Both literatures have evidential significance, but the $\mathrm{p}$-curve of negative $\mathrm{x}$-phi is less indicative of $\mathrm{p}$-hacking than that of positive x-phi (Figures 9 and 10). This difference may be explained by the sample sizes of studies in negative and positive x-phi, rather than any difference in the nature of these studies, 
their targets, or their implications. Unsurprisingly, studies with fewer participants (fewer than 20) look to be p-hacked, although they still have evidential value (Figure 8). ${ }^{1}$

We found no difference between the studies conducted solely by philosophers and those co-authored with non-philosophers (Figure 7). This undermines the suggestion that experimental philosophers are likely to engage in questionable research practices or more generally to engage in poor psychology because of their lack of training in psychology (Williamson 2010).

Currently there are two main limitations of the p-curve test. First, it does not work with discrete test statistics (e.g., difference of proportions tests). We had 83 instances of such tests in our data set, although only 19 of these were main results (out of a total of 1030 main results). So this is not a serious issue. Second, the p-curve is slightly 'pessimistic,' in the sense that it is more likely to present corpora as lacking evidential significance than having it (Simonsohn, Nelson, and Simmons 2014a: 546). We were happy to adopt this pessimistic approach, but since each pcurve displayed evidential value, it has not been a concern.

\section{Conclusion}

Our investigation of the evidential value and presence of questionable research practices in x-phi led us to develop a corpus of studies and perform p-curve analyses on them. Our findings indicate that, both as a whole and within all subsets, the corpus has evidential value. P-hacking has probably occurred in a few areas of x-phi, in particular, during its first years, even if the effects reported are, on the whole, genuine. We hope to see $\mathrm{x}$-phi continue the trajectory of

\footnotetext{
${ }^{1}$ We also found no difference in articles compared to book chapters ( $p$-curves not reported for considerations of space).
} 
methodological progress we have identified. Hopefully, the field will produce even better studies and eliminate questionable research practices from its toolbox.

\section{Funding}

This work was supported by the University of Pittsburgh's Center for Philosophy of Science and the Social Sciences and Humanities Research Council of Canada.

\section{References}

Adleberg, T., Thompson, M., and Nahmias, E. 2015. Do men and women have different philosophical intuitions? Further data. Philosophical Psychology 28: 615-641.

Bakker, M. and Wicherts, J. M. 2011. The (mis)reporting of statistical results in psychology journals. Behavior research methods 43: 666-678.

Cappelen, H. 2012. Philosophy without Intuitions. Oxford: Oxford University Press.

Colaço, D., Kneer, M., Alexander, J. and Machery, E. Manuscript. On second thought: A refutation of the reflective judgment defense.

Colaço, D. and Machery, E. 2017. The intuitive is a red herring. Inquiry 60: 403-419.

Colombo M., Duev G. and Nuijten M.B., and Sprenger, J. 2018 Statistical reporting inconsistencies in experimental philosophy. PLoS ONE 13: e0194360. https://doi.org/10.1371/journal.pone.0194360.

Cova, F., Abatista, A. G. F., Allard, A., Andow, J., Attie, M., Beebe, J., ... Strickland, B. 2018. The XPhi Replicability Project. http://doi.org/10.17605/OSF.IO/DVKPR.

Cullen, S. 2010. Survey-driven romanticism. Review of Philosophy and Psychology 1: 275-296. 
Deutsch, M. E. 2015. The Myth of the Intuitive: Experimental Philosophy and Philosophical Method. Cambridge: MIT Press.

Head, M. L., Holman, L. Lanfear, R., Kahn, A. T., and Jennions, M. D. 2015. The extent and consequences of p-hacking in science. PLOS Biology DOI:10.1371/journal.pbio.1002106.

Kauppinen, A. 2007. The rise and fall of experimental philosophy. Philosophical explorations, $10,95-118$

Kim, M., and Yuan, Y. 2015. No cross-cultural differences in the Gettier car case intuition: A replication study of Weinberg et al. 2001. Episteme 12: 355-361.

Ludwig, K. 2007. The epistemology of thought experiments: First person versus third person approaches. Midwest Studies in Philosophy 31: 128-159.

Ludwig, K. 2018. Thought experiments and experimental philosophy. In The Routledge Companion to Thought Experiments, ed. M. Stuart, Y. Fehige and J. Brown, 385-405. London: Routledge.

Machery, E. 2017. Philosophy within its Proper Bounds. Oxford: Oxford University Press.

Machery, E., Stich, S. P., Rose, D., Chatterjee, A., Karasawa, K., Struchiner, N., Sirker, S., Usui, N. and Hashimoto, T. Forthcoming. Gettier across cultures. Nous doi: 10.1111/nous. 12110 .

O’Neill, E. and Machery, E. 2014. Experimental philosophy: What is it good for? In Current controversies in experimental philosophy, eds. E. Machery and E. O’Neill, vii-xxix. New York: Routledge.

Mahowald, K., James, A., Futrell, R. and Gibson, E. 2016. A meta-analysis of syntactic priming in language production. Journal of Memory and Language 91: 5-27. 
Medina, J., and Cason, S. 2017. No evidential value in samples of transcranial direct current stimulation (tDCS) studies of cognition and working memory in healthy populations. Cortex 94: 131-141.

Nuijten, M. B., Hartgerink, C. H., van Assen, M. A., Epskamp, S. and Wicherts, J. M. 2016. The prevalence of statistical reporting errors in psychology (1985-2013). Behavior research methods 48: 1205-1226.

Scholl, B. ms. Two kinds of experimental philosophy (and their experimental dangers).

Segerstrom, S.C. and Miller, G.E. Applying the p curve to PNI: Are effects of stress on immunity real or incidental? Brain, Behavior, and Immunity 32: e5.

Seyedsayamdost, H. 2015a. On normativity and epistemic intuitions: Failure of replication. Episteme 12: 95-116.

Seyedsayamdost, H. 2015b. On gender and philosophical intuition: Failure of replication and other negative results. Philosophical Psychology 28: 642-673.

Simonsohn, U., Nelson, L. D. and Simmons, J. P. 2014a. P-curve: A key to the file-drawer. Journal of Experimental Psychology: General 143: 534-547.

Simonsohn, U., Nelson, L. D. and Simmons, J. P. 2014b. P-curve and effect size correcting for publication bias using only significant results. Perspectives on Psychological Science 9: $666-681$

Simonsohn, U., Simmons, J. P. and Nelson, L. D. 2015. Better p-curves: Making p-curve analysis more robust to errors, fraud, and ambitious p-hacking, a reply to Ulrich and Miller. Journal of Experimental Psychology: General 144: 1146-1152.

Simmons, J. P. and Simonsohn, U. 2017. Power posing: P-curving the evidence. Psychological science 28: 687-693. 
Stich, S. and Tobia, K. 2018. Intuition and its critics. In The Routledge Companion to Thought Experiments, ed. M. Stuart, Y. Fehige and J. Brown, 369-384. London: Routledge.

Stuart, M. 2015. Philosophical conceptual analysis as an experimental method. In Meaning, Frames and Conceptual Representation, eds. T. Gamerschlag, D. Gerland, R. Osswald and W. Petersen, 267-292. Düsseldorf: Düsseldorf University Press.

Vadillo, M. A., Gold, N. and Osman, M. 2016. The bitter truth about sugar and willpower: The limited evidential value of the glucose model of ego depletion. Psychological Science 27: $1207-1214$.

Vazire, S. 2015. Experimental philosophy: The view from social/personality psychology. Annals of the Japan Association for Philosophy of Science 23: 45-52.

Williamson, T. 2010. Philosophy vs. imitation psychology. New York Times, 2010/08/19, available at http://www.nytimes.com/roomfordebate/2010/08/19/x-phis-new-take-on-oldproblems/philosophy-vs-imitation-psychology?

Woolfolk, R. L. 2011. Empirical tests of philosophical intuitions. Consciousness and Cognition, 20: $415-416$.

Woolfolk, R. L. 2013. Experimental philosophy: A methodological critique. Metaphilosophy 44: $79-87$. 